ORIGINAL ARTICLE

\title{
A Randomized Controlled Trial Study on the Effect of Adding Dexmedetomidine to Bupivacaine in Supraclavicular Block Using Ultrasound Guidance
}

\author{
Nazia Nazir ${ }^{1}$, Shruti Jain ${ }^{1}$
}

ABSTRACT

BACKGROUND: The benefits of regional anesthetic techniques are well established. Use of additives to local anesthetics can prolong these benefits. The aim of this study was to find out the effect of adding dexmedetomidine to bupivacaine for supraclavicular block.

METHODS: In this randomized, double-blind study, 70 ASA I \& II patients of either sex undergoing elective surgeries on the upper limb were given supraclavicular block under ultrasound guidance. Group $C(n=35)$ received $38 \mathrm{~mL} 0.25 \%$ bupivacaine $+2 \mathrm{~mL}$ normal saline and group $D$ received $38 \mathrm{~mL} 0.25 \%$ bupivacaine $+1 \mu \mathrm{g} / \mathrm{kg}$ dexmedetomidine $(2 \mathrm{~mL})$. Patients were observed for, onset of motor and sensory block, duration of motor and sensory block, duration of analgesia, sedation score, hemodynamic changes and any adverse events.

RESULTS: In group $D$, the onset was faster $(P<0.001)$, durations of sensory and-motor block duration $\theta f$ and analgesia were prolonged as compared to group $C(P<0.0001)$.There was a significant drop in heart rate $(H R)$ from the baseline in group $D(P<0.05)$ at 30, 60, 90 and 120 min. However, none of the patients dropped HR below 50/min. Mean Arterial Pressure (MAP) remained unaffected. The patients in group $D$ were more effectively sedated than those in group $C(P<0.05)$. No adverse event was reported in either group.

CONCLUSION: Dexmedetomidine as adjuvant to bupivacaine in supraclavicular block resulted in faster action, prolonged motor and sensory block, prolonged analgesia with hemodynamic stability and adequate sedation.

KEYWORDS: Dexmedetomidine, analgesia, supraclavicular block

DOI: http://dx.doi.org/10.4314/ejhs.v26i6.9

\section{INTRODUCTION}

Surgeries of the upper limb are usually done under brachial plexus block. Various agents have been tried as adjuvant to increase the duration of post operative analgesia provided by local anesthetics (LA).We tried dexmedetomidine, an $\alpha 2$ adrenergic agent, as an adjuvant to bupivacaine as it has sedative, analgesic, sympatholytic and cardiovascular stabilizing effects. The aim of this study was to compare the postoperative analgesic efficacy and the safety of dexmedetomidine for brachial plexus blockade along with bupivacaine.

\section{METHODS}

After approval from Hospital Ethical Committee, a randomized, double-blind, prospective clinical study was initiated. Seventy ASA Grade I and II patients of either sex, aged 18-60 years, weighing 30-65 kg undergoing upper limb surgeries were selected. Patients with known hypersensitivity to LA, hypertension, diabetes mellitus, bleeding disorders, cardiac, liver or renal disease, pregnant women, infection at the site of injection and preexisting peripheral neuropathy were excluded from the study. The patients were divided in two groups of 35 patients each on the basis of adjunct given to them. The randomization was achieved

\footnotetext{
${ }^{1}$ Department of Anesthesiology, School of Medical Sciences \& Research, Sharda University, Greater Noida Corresponding Author: Nazia Nazir, Email: nazunazir@ gmail.com
} 
by random number table using a sealed envelope technique. The drug solutions were prepared by an anesthesiologist who was not involved in the study. The two groups were:

- Group C: control group; received injection bupivacaine $(0.25 \%) 38 \mathrm{~mL}$ plus $2 \mathrm{~mL}$ normal saline, and

- Group D: study group; received injection bupivacaine $(0.25 \%) 38 \mathrm{~mL}$ plus dexmedetomidine $1 \mu \mathrm{g} / \mathrm{kg}$ diluted to $2 \mathrm{~mL}$.

Prior to surgery, all patients were fasted for six hours. Baseline heart rate, blood pressure and oxygen saturation were recorded. In the unaffected arm, after securing $18 \mathrm{G}$ intravenous cannula, ringer's lactate was started. Premedication with anti-anxiety and sedative drugs was not given. All the patients received brachial plexus block through the supraclavicular approach by an experienced anesthesiologist under ultrasound (US) guidance (Sonosite Titan $^{\mathrm{TM}}$ TM US machine with a 6-13 $\mathrm{MHz}$ linear probe). After the brachial plexus was identified, the needle was introduced under US guidance. Correct position of the needle was confirmed by injecting saline boluses $(0.5 \mathrm{~mL})$ before drug injection. After negative aspiration, $40 \mathrm{~mL}$ of drug solution was injected.

Patients were assessed by a different anesthetist for hemodynamic changes, onset of motor and sensory blockade, duration of motor and sensory block, duration of analgesia, sedation score, and any adverse events. Both anesthetists were blinded to the treatment groups. Monitoring of vital parameters (HR and MAP) was done every five minutes for the first 30 minutes and thereafter every 10 minutes till the end of surgery and every 60 minutes(min) post-operatively. Bradycardia was defined as HR less than 50 beats/min which was treated with inj. atropine $0.6 \mathrm{mg}$ i.v.

Sensory block was assessed by the pin prick method at every three minute after drug injection by three point scale. According to this scale, (1) grade 0 was no block, grade 1 was sensory blockade with persistence of touch and grade 2 was complete sensory blockade. Onset of sensory block was the time from drug injection to grade 2 block. The duration of sensory block was the time from drug injection to the complete resolution of anesthesia.

Assessment of motor block was done using modified Bromage scale (2) by the same observer at every three minute until complete motor blockade. According to this scale, grade 0 was free movement of elbow, wrist and fingers, grade 1 was inability to extend the arm for 2 second, grade 2 was inability to extend the arm or flex the forearm, and grade 3 was inability to extend the arm, flex the forearm or move the fingers. Motor blockade of elbow was checked only in those cases in which it was possible; otherwise, only the movement of fingers was assessed.

The onset of motor block was the time from drug injection to grade 3 block. The duration of motor block was the time from drug injection to the complete recovery of motor function of the hand and forearm. The block was considered incomplete when any of the segments supplied by median, radial, ulnar and musculocutaneous nerve did not have complete sensory or motor block even after 30 minutes of drug injection. These patients were then excluded from the study and were given general anesthesia. For assessment of sedation of patients, Ramsay Sedation Score (3) was used.

Grade 1: Patient anxious and agitated or restless, or both

Grade 2: Patient cooperative, oriented and tranquil Grade 3: Patient responds to commands only

Grade 4: Patient exhibits brisk response to light glabellar tap or loud auditory stimulus

Grade 5: Patient exhibits a sluggish response to light glabellar tap or loud auditory stimulus

Grade 6: Patient exhibits no response.

The assessment of postoperative pain was done hourly in the recovery room and in surgical ward with the help of Numeric Rating Scale (1-10) (4). Zero was considered as no pain, 1-3 as mild pain, 4-6 as moderate pain and $7-10$ as severe pain. At score of 4, rescue analgesic (inj. diclofenac sodium $(1.5 \mathrm{mg} / \mathrm{kg})$ intramuscularly) was given. Duration of analgesia was the time from drug injection to the time of first request of analgesia. An attending nurse was advised to repeat Inj. diclofenac $1.5 \mathrm{mg} / \mathrm{kg}$ every eight hours thereafter. All patients were observed for any sideeffects like nausea, vomiting, dryness of mouth, pneumothorax, hematoma, local anesthetic toxicity and post-block neuropathy in the intraand postoperative periods.

Keeping the time of first analgesic request as primary variable, sample size was calculated. A pilot study with five patients in each group was conducted. Presuming the difference in 
the time of first analgesic request and effect size obtained to be true, we calculated that 35 patients would be required in each group for the study with power 0.8 and significance of 0.05.The data was coded, entered and analysed using SPSS (standard statistical software SPSS) software Inc., version 16.0 for windows. Data was summarized using mean, standard deviation, minimum and maximum for quantitative variables and relative frequencies (percentages) for categorical variables. Normally distributed variables between the two groups were analysed using t-tests. Non-normally distributed variables were analysed using Mann Whitney test. Categorical variables were analysed using the Pearson's Chi-square $(\chi 2)$ test. Fisher exact test was used instead when the expected frequency is less than $5 . \mathrm{P}<0.05$ was considered as statistically significant and $\mathrm{P}<0.001$ as highly significant.

\section{RESULTS}

The patients in both groups were comparable with respect to the demographic parameters. The baseline hemodynamic parameters were comparable in both groups. In group D, HR was significantly decreased from the baseline at 30,60, 90 and 120 minutes as compared to group C (Figure 1) $(\mathrm{P}<0.001)$ but none of the patients developed bradycardia. No statistical difference was observed on comparison of mean arterial pressures (MAP) between the two groups (Figure 2) $(\mathrm{P}>0.05)$.

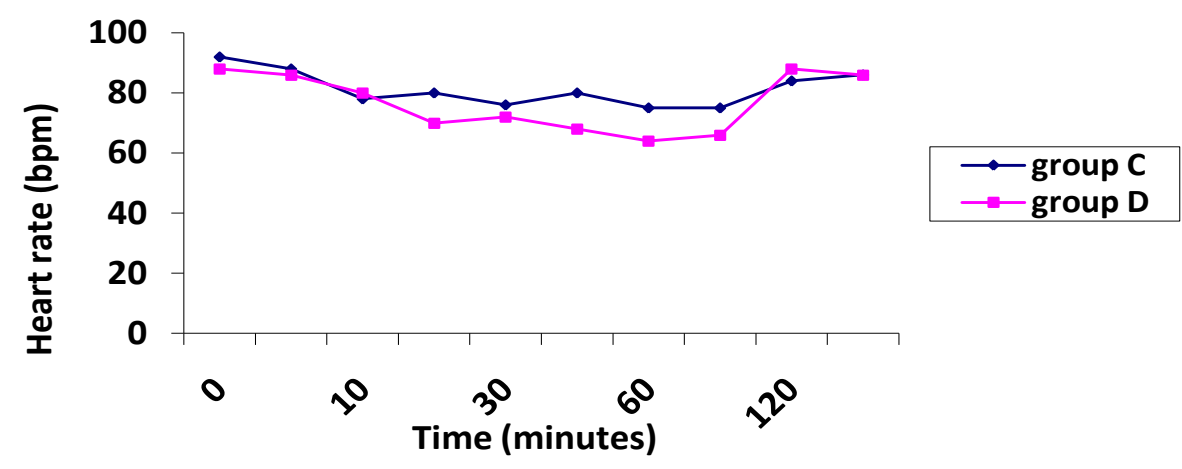

Figure 1: Comparison of heart rates between groups

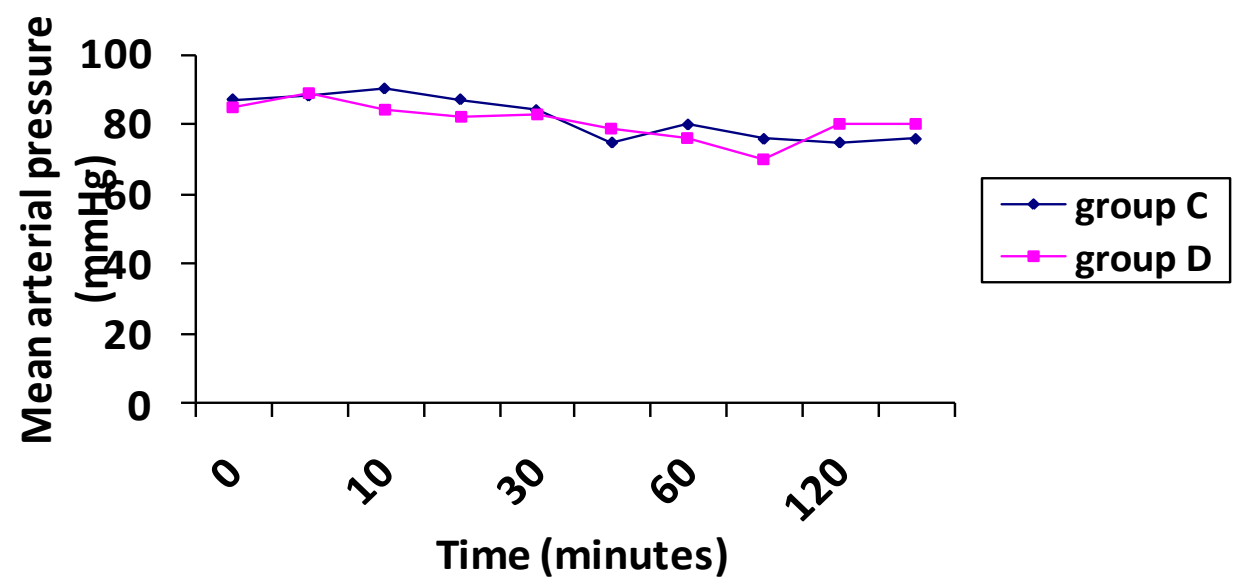

Figure 2: Comparison of Mean arterial pressures between groups 
The onset of sensory and motor block was faster in group $\mathrm{D}$ as compared to group $\mathrm{C}(\mathrm{P}<0.001)$ (Table 1). The duration of sensory and motor blockade was significantly prolonged in group D (P <0.0001) (Table 1). Similarly, the duration of analgesia was also significantly prolonged in group $\mathrm{D}$ as compared to group $\mathrm{C}(\mathrm{P}<0.0001)$
(Table 1). In group $\mathrm{D}$, none of the patients was anxious. RSS 2 was seen in $83 \%$ of patients of group D while in group C, $85 \%$ of the patients had sedation score of 1 (Table 3) No adverse effects of drugs or complications of the technique were observed in any group.

Table 1: Demographic characteristics

\begin{tabular}{lll}
\hline Demographic parameters & Group C $(\mathrm{n}=35)$ & Group D $(\mathrm{n}=35)$ \\
& Mean \pm S.D & Mean \pm S.D \\
\hline Age (years) & $38 \pm 10.9$ & $37.6 \pm 9.3$ \\
Weight (kilograms) & $50 \pm 8.9$ & $52 \pm 8.7$ \\
Gender (Male/ Female) & $17 / 18$ & $20 / 15$ \\
\hline
\end{tabular}

Table 2: Onset, duration of sensory \& motor block \& duration of analgesia

\begin{tabular}{lclll}
\hline Parameter & $\begin{array}{l}\text { Group C }(\mathrm{n}=35) \\
\text { Mean } \pm \text { S.D }\end{array}$ & $\begin{array}{l}\text { Group D }(\mathrm{n}=35) \\
\text { Mean } \pm \text { S.D }\end{array}$ & t- value & P-value \\
\hline Onset of sensory block ( min) & $14.76 \pm 3.24$ & $12.24 \pm 2.8$ & 3.48 & 0.001 \\
Onset of motor block (min) & $20.53 \pm 2.43$ & $15.8 \pm 1.9$ & 9.07 & 0.0001 \\
Duration of sensory block (min) & $200 \pm 33.85$ & $698 \pm 43.9$ & 53.14 & 0.0001 \\
Duration of motor block (min) & $188 \pm 28.67$ & $673 \pm 79.46$ & 33.96 & 0.0001 \\
Duration of analgesia (min) & $210 \pm 35.88$ & $722 \pm 88.45$ & 31.73 & 0.0001 \\
\hline
\end{tabular}

Table 3: Comparison of Sedation scores

\begin{tabular}{lll}
\hline Sedation score (RSS) & Group C $(\mathrm{n}=35)$ & Group D $(\mathrm{n}=35)$ \\
\hline 1 & $30(85 \%)$ & 0 \\
2 & $5(15)$ & $29(83 \%)$ \\
3 & - & $6(17 \%)$ \\
4 & - & - \\
5 & - & - \\
$\mathbf{6}$ & - & - \\
\hline
\end{tabular}

RSS= Ramsay sedation score

\section{DISCUSSION}

Dexmedetomidine, a highly selective alpha-2 agonist, has sedative, analgesic, sympatholytic and cardiovascular stabilizing effects (5). It causes significant opioid sparing as well as a decreased requirement of inhalational agents during anesthesia. (6) Various studies have shown that dexmedetomidine when added to LA in regional blocks (spinal, (7) epidural,(8) caudal, $(9,10)$ axillary,(11) supraclavicular,(12) Bier's(13) and greater palatine nerve block(14)) causes prolongation of duration of block and postoperative analgesia.

The highly selective action of dexmedetomidine on alpha-2 adrenoreceptors $(\alpha 2: \alpha 1=1620: 1)$ results in sedation and analgesia without unwanted vascular effects from activation of alpha1receptors. In addition, a reversal drug (Atipamezole) for the sedative effect of dexmedetomidine is also available. These properties make dexmedetomidine a suitable agent for sedation and analgesia during the perioperative period. (15) Dose of $1 \mu \mathrm{g} / \mathrm{kg}$ of dexmedetomidine was used for supra clavicular block in our study. 
Similar dose were also used in previous studies. $(11,12)$ The use of ultrasound gave us the advantage of real time monitoring of drug injection around the plexus. However, the volume of bupivacaine used was not decreased because volume rather than the concentration of LA was important to achieve an effective nerve blockade. (16)

Significant drop in HR from the baseline was observed in patients of group D. However, none of the patients developed bradycardia or hypotension. The reduction in HR occurred due to the sympatholytic effect of alpha-2 agonists (11). Various studies which used dexmedetomidine in dose similar to our study, few of them had reported cases of bradycardia requiring treatment, $(11,17,18)$ while others had not reported any incidence of bradycardia (12).

The onset of sensory and motor blockade was faster in the group receiving dexmedetomidine than in the control group. The faster onset could be due to local action of dexmedetomidine on nerve compound action potential (19) as well as enhancement of anesthetic action of LA (20). The results were in agreement with previous studies in which dexmedetomidine was added to bupivacaine in supraclavicular block $(11,12,18,21)$.

The duration of motor and sensory block as well as post operative analgesia was prolonged in group D. The result can be explained by peripheral and central actions of dexmedetomidine. Peripherally, it produces analgesia by decreasing the release of norepinephrine which causes inhibition of on nerve action potentials. Centrally, it causes inhibition of the release of substance $\mathrm{P}$ in the nociceptive pathway at the level of the dorsal root neuron which produces analgesia (11).

The patients in group D were comfortable and did not require any sedative. This was due to the sedative effect of dexmedetomidine by virtue of its action on the subtype $\mathrm{A}$ and $\mathrm{C}$ of alpha-2 adrenoreceptors in the CNS (locus coerulus) which results in sedation, analgesia and anxiolysis. (15)

In conclusion, dexmedetomidine is an effective adjuvant to bupivacaine for supraclavicular block. It results in faster onset of block with prolonged duration of analgesia, effective sedation and stable hemodynamics

\section{REFERENCES}

1. Eledjam J.J, Deschodtt J, Viel EJ, et al. Brachial plexus block with bupivacaine: effects of added alpha- adrenergic agonists: comparison between clonidine and epinephrine. Can J Anaesth. 1991; 38(7), 8705.

2. Bromage PR. A comparison of hydrochloride and carbon dioxide salts of lidocaine and prilocaine in epidural analgesia. Acta Anaesthesiol. Scand. Suppl. 1965; 16: 55-69.

3. Ramsay MA, Savage TM, Simpson BR, Godwin R. Controlled sedation with alphaxolone-alphadolone. $\mathrm{Br} \quad \mathrm{Med} \quad J .1974$; 2:656-9.

4. Krebs EE, Carey TS, Weinberger M. Accuracy of the Pain Numeric Rating Scale as a Screening Test in Primary Care. J Gen Intern Med. 2007; 22(10): 1453-8.

5. Arain SR, Ebert TJ. The efficacy, side effects, and recovery characteristics of dexmedetomidine versus propofol when used for intraoperative sedation. Anesth Analg. 2002; 95(2):461-6.

6. Keniya VM, Ladi S, Naphade R. Dexmedetomidine attenuates sympathoadrenal response to tracheal intubation and reduces perioperative anaesthetic requirement. Indian J Anaesth. 2011; 55:352-7.

7. Kanazi GE, Aouad MT, JAbbour- Khoury SL, et al. Effects of low dose Dexmedetomidine or clonidine on characteristics of spinal block. Acta Anaesthesiol Scand. 2006; 50:222-7.

8. Bajwa SJ, Bajwa SK, Kaur J, Singh G, Arora $\forall$, Gupta S, et al. Dexmedetomidine and clonidine in epidural anaesthesia: A comparative evaluation. Indian Jr Anaesth. 2011; 55(2): 116-121.

9. Anand VG, Kannan M, Thavamani A, Bridgit MJ. Effects of dexmedetomidine added to caudal ropivacaine in paediatric lower abdominal surgeries. Indian J Anaesth. 2011; 55(4): 340-6.

10. El Shamaa HA, Ibrahim M. A comparative study of the effect of caudal dexmedetomidine versus morphine added to bupivacaine in pediatric infra-umbilical surgery. Saudi $J$ Anaesth. 2014; 8(2): 155-160. 
11. Esmaoglu A, Yegenoglu F, Akin A, Turk CY. Dexmedetomidine added to levobupivacaine prolongs axillary brachial plexus block. Anaesth Analg.2010; 111:1548-51.

12. Swami SS, Keniya VM, Ladi SD, Rao R. Comparison of dexmedetomidine and clonidine ( $\alpha 2$ agonist drugs) as an adjuvant to local anaesthesia in supraclavicular brachial plexus block: A randomised double-blind prospective study. Indian journal of anaesthesia. 2012; 56(3): 243-9.

13. Memis D, Turan A, Karamanlioglu B, Pamukçu Z, Kurt I. Adding dexmedetomidine to lignocaine for IVRA. Anesth Analg. 2004; 98:835-40.

14. Obayah GM, Refaie A, Aboushanab O, Ibraheem $\mathrm{N}$, Abdelazees $\mathrm{M}$. Addition of dexmedetomidine to Bupivacaine for greater palatine nerve block prolongs postoperative analgesia after cleft palate repair. Eur $J$ Anaesthesiol. 2010; 27:280-4.

15. Gertle R, Brown C, Mitchell D, Silvius E. Dexmedetomidine: a novel sedative-analgesic agent. Proc (Bayl Univ Med Cent). 2001; 14(1): 13-21.

16. Duggan E1, El Beheiry H, Perlas A, et al. Minimum effective volume of local anesthetic for ultrasound-guided supraclavicular brachial plexus block. Reg Anesth Pain Med. 2009; 34(3):215-8.
17. Das A, Majumdar S, Halder S, et al. Effect of dexmedetomidine as adjuvant in ropivacaineinduced supraclavicular brachial plexus block: A prospective, double-blinded and randomized controlled study. Saudi $J$ of Anesth. 2014; 8 (5): 72-7

18. Agarwal S, Aggarwal R, Gupta P. Dexmedetomidine prolongs the effect of bupivacaine in supraclavicular brachial plexus block. J Anaesthesiol Clin Pharmacol 2014; 30(1): 36-40.

19. Kosugi T, Mizuta K, Fujita T, Nakashima M, Kumamoto E. High concentration of dexmedetomidine inhibits compound action potential in frog sciatic nerve without adrenoreceptor activation. $\mathrm{Br} \quad J$ Pharmacol.2010; 160: 1662-1676.

20. Yoshitomi T, Kohjitani A, Maeda S, Higuchi $\mathrm{H}$, Shimada M, Miyawaki $\mathrm{T}$. Dexmedetomidine enhances the local anesthetic action of lidocaine via an alpha-2A adrenoceptor. Anesth Analg. 2008; 107(1):96101.

21. Gandhi R, Shah A, Patel I. Use of dexmedetomidine along with bupivacaine for brachial plexus block. National Journal of Medical Research. 2012; 2(1):67-69. 